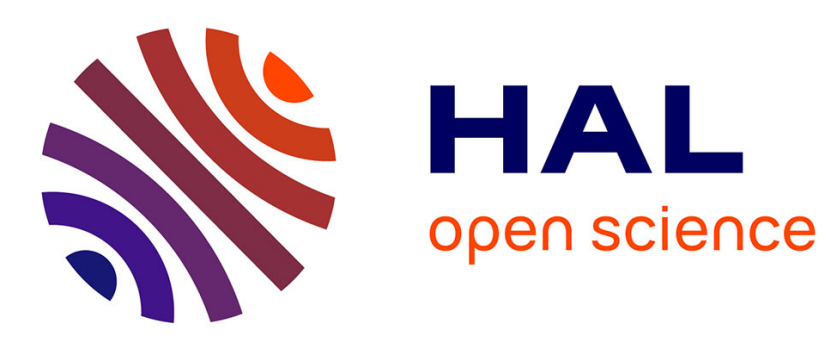

\title{
Sensitivity of 2-D turbulent flow past a D-shaped cylinder using global stability
}

Philippe Meliga, Gregory Pujals, Eric Serre

\section{To cite this version:}

Philippe Meliga, Gregory Pujals, Eric Serre. Sensitivity of 2-D turbulent flow past a D-shaped cylinder using global stability. Physics of Fluids, 2012, 24, pp.061701. 10.1063/1.4724211 . hal-01061797

\section{HAL Id: hal-01061797 \\ https://hal.science/hal-01061797}

Submitted on 8 Sep 2014

HAL is a multi-disciplinary open access archive for the deposit and dissemination of scientific research documents, whether they are published or not. The documents may come from teaching and research institutions in France or abroad, or from public or private research centers.
L'archive ouverte pluridisciplinaire HAL, est destinée au dépôt et à la diffusion de documents scientifiques de niveau recherche, publiés ou non, émanant des établissements d'enseignement et de recherche français ou étrangers, des laboratoires publics ou privés. 


\title{
Sensitivity of 2-D turbulent flow past a D-shaped cylinder using global stability
}

\author{
Philippe Meliga, ${ }^{1}$ Gregory Pujals, ${ }^{2}$ and Éric Serre ${ }^{1}$ \\ ${ }^{1}$ M2P2, CNRS-Universités d'Aix-Marseille, 13451 Marseilles, France \\ ${ }^{2}$ PSA Peugeot Citroën, Centre Technique de Velizy, 78943 Vélizy-Villacoublay Cedex, France
}

(Received 24 January 2012; accepted 9 May 2012; published online 5 June 2012)

\begin{abstract}
We use adjoint-based gradients to analyze the sensitivity of turbulent wake past a D-shaped cylinder at $R e=13000$. We assess the ability of a much smaller control cylinder in altering the shedding frequency, as predicted by the eigenfrequency of the most unstable global mode to the mean flow. This allows performing beforehand identification of the sensitive regions, i.e., without computing the actually controlled states. Our results obtained in the frame of 2-D, unsteady Reynolds-averaged NavierStokes compare favorably with experimental data reported by Parezanović and Cadot [J. Fluid Mech. 693, 115 (2012)] and suggest that the control cylinder acts primarily through a local modification of the mean flow profiles. (일 2012 American Institute of Physics. [http://dx.doi.org/10.1063/1.4724211]
\end{abstract}

The seminal analysis of Strykowski and Sreenivasan ${ }^{1}$ provides experimental evidence that a small control cylinder suitably positioned in the wake of a main cylinder can alter vortex shedding at Reynolds numbers closely above the instability threshold, either by suppressing the shedding, or by decreasing its frequency. For various diameter ratios of the two cylinders, the authors measured the influence of the control cylinder in terms of sensitivity maps showing the regions around the main cylinder where shedding was most affected. This finding was revisited by $\mathrm{Hill}^{2}$ in the limit of small cylinder ratios, i.e., for control cylinders much smaller than the main cylinder. Hill took advantage of the adjoint method to estimate the sensitivity of the natural shedding mode, i.e., the global instability mode correlated to the uncontrolled shedding activity. He modeled the effect of the control cylinder by a supply of momentum to the flow equal and opposite to the anticipated drag, and thereby retrieved the structure of the experimental sensitivity maps beforehand, without ever having calculated the actual controlled states. For such flow dominated by an instability mechanism, the approach offers an attractive alternative to bottleneck "trial and error" procedures in so far as it allows exhaustive coverage of large parameter spaces (position and diameter of the cylinder) at very low computational costs.

Although the early theoretical concepts have risen for more than 50 years, the development of predictive tools capable of gaining valuable information regarding the most sensitive regions of laminar flows for open-loop control based on the underlying physics is a recent achievement. ${ }^{3-7}$ The present research is an attempt at pushing forward the development of the method in the context of turbulent flows, for which successful experimental implementation of the control cylinder technique is also reported. ${ }^{8}$ It puts emphasis on the role of the time-averaged mean flow in turbulent wakes at Reynolds numbers of order $10^{4}$, hence dominated by the shedding of large-scale vortices. The line of thought is as follows:

1. We perform a global linear stability analysis of the mean flow, as in Refs. 9-13. By doing so, we identify the natural shedding mode, whose eigenfrequency turns to be a good predictor of the nonlinear shedding frequency,

2. We generalize Hill's analysis and assess the sensitivity of the shedding mode with respect to a small control cylinder. By doing so, we identify specific regions where the control cylinder yields either an increase or a decrease of the eigenfrequency. 
3. The relevance of the approach for our case is assessed by the close agreement between the obtained sensitivity map and experimental results reported recently in the literature by Parezanović and Cadot. ${ }^{14}$

Two-dimensional (2-D) numerical simulations are undertaken in the frame of unsteady Reynolds-averaged Navier-Stokes (RANS), whose ability in predicting the basic mean flow features and turbulence statistics of those flow cases considered herein is carefully assessed in Refs. 15 and 16. A good agreement is evidenced with regards to existing experimental and numerical data, which can be ascribed to the fact that unlike inherently unsteady turbulent flows, there is a clear separation between the large-scale vortices shed in the wake and the small-scale Kelvin-Helmholtz-like instability developing in the shear layers and further yielding the production of turbulence by selective amplification of the background noise. In practice, large-scales are thus resolved by time-integration while small-scales are modeled to provide closure for the averaged Reynolds stresses. We use the standard form of the one-equation Spalart-Allmaras turbulence model. ${ }^{17}$ The flow motion is thus described by the 2-D velocity field $\boldsymbol{u}=(u, v)^{\mathrm{T}}$ of components $u$ and $v$ in the streamwise $x$ and transverse $y$ directions, the pressure $p$, and the turbulence field variable $\tilde{v}$ physically related to the eddy viscosity, denoted the Spalart-Allmaras (SA) variable. We write here the governing equations in terms of the state vector $\boldsymbol{q}=(\boldsymbol{u}, p, \tilde{v})^{\mathrm{T}}$ as

$$
\boldsymbol{B} \partial_{t} \boldsymbol{q}+\boldsymbol{M q}+\boldsymbol{N}(\boldsymbol{q}, \boldsymbol{q})=\mathbf{0},
$$

where $\boldsymbol{M}$ and $\boldsymbol{B}$ are two linear differential operators and $\boldsymbol{N}$ contains all remaining nonlinear terms. For the sake of brevity, the detailed equations, as well as all relevant information about the turbulence modeling constants and functions, are supplied as supplementary material. ${ }^{18}$ Our focus is on flows that can be treated as fully turbulent, which removes the need for a trip-term, i.e., an additional source term in the right-hand side of the transport equation for $\tilde{v}$ meant to force the transition at a prescribed location. The numerics rely on the incompressible, finite-elements solver presented in Ref. 19 in which the turbulence model has been implemented via user-defined functions. For time discretization, we use a first-order time scheme based on characteristics methods. For spatial discretization, we use Arnold-Brezzi-Fortin MINI-elements (piecewise linear continuous finite elements with three degrees of freedom at each triangle edge for pressure, same element enriched with a cubic bubble function at the barycenter of each triangle for both velocity components and the SA variable). The time-averaged mean flow, denoted in the following by an overbar, is obtained from a random initial condition, first advanced in time until coherent vortex shedding shows up, then averaged on the fly until the relative variation in the mean energy per grid point is less than $10^{-8}$. In practice, this takes roughly 3 flow-through times and subsequently 300 shedding cycles, independently of the flow configuration discussed in the following.

For validation purposes of our numerical solver, we consider first the widely benchmarked turbulent flow past a square cylinder at the Reynolds number $\operatorname{Re}=U_{\infty} D / v=22000$, where $U_{\infty}$ is the inflow velocity, $D$ is the cylinder diameter, and $v$ is the kinematic viscosity. Typical timestep employed is $\Delta t=0.1$, which allows sampling $\sim 80$ time-steps over a shedding period and converging force coefficients in terms of mean and root mean square (rms) values. ${ }^{16}$ The size of the computational domain is that recommended by Rodi, ${ }^{15}$ along with strong clustering close to the walls to capture the near-wall turbulent regions. Symmetric conditions are applied at the upper and lower boundaries, no-slip conditions at the wall, and advective conditions at the outlet. A unit streamwise velocity is imposed at the inlet, where turbulence is forced prescribing the ratio of the eddy to the kinematic viscosity. Varying the value of this ratio was found to have little influence on the numerical results. Our reference simulation is for a mesh $M_{1}$ built from 144258 triangles, hence resulting in 723570 degrees of freedom. Results reported in Table I compare well to 2-D RANS numerical data available from the literature. A similar agreement exists with three-dimensional (3-D) data reported from experiments and from spanwise-averaged large-eddy simulations (LES) performed with span $H=4 D$, hence suggesting that the 2-D flow assumption holds true. Grid independence has been checked comparing to a higher resolution simulation with mesh $\mathrm{M}_{2}$ built from 381406 triangles (1910790 degrees of freedom), all numerical values being found to change by less by $4 \%$. 
TABLE I. Synoptic table of representative mean and fluctuating quantities for flow past a square cylinder. $\bar{l}_{R}$ : mean recirculation length measured from the rear surface, $\bar{C}_{D}$ : mean drag coefficient, $C_{D^{\prime}}$ and $C_{L^{\prime}}$ : rms of drag and lift coefficients, St: Strouhal number.

\begin{tabular}{|c|c|c|c|c|c|c|}
\hline Contribution & Model & $\bar{l}_{R}$ & $\bar{C}_{D}$ & $C_{D^{\prime}}$ & $C_{L^{\prime}}$ & $S t$ \\
\hline Lyn et al. ${ }^{20}$ & 3-D Exp. & 0.88 & 2.1 & $\ldots$ & $\ldots$ & 0.132 \\
\hline $\mathrm{Lee}^{21}$ & 3-D Exp. & $\ldots$ & 2.05 & $0.16-0.23$ & $\ldots$ & $\ldots$ \\
\hline Rodi et al.22 a & 3-D LES & 0.82 & 2.2 & 0.14 & 1.01 & 0.13 \\
\hline Minguez et l. $^{23}$ a & 3-D LES & 0.77 & 2.2 & $\ldots$ & $\ldots$ & 0.141 \\
\hline Rodi et al..$^{22 \mathrm{~b}}$ & 2-D RANS, 2-layer $k$ - $\omega$ & 0.75 & 2.004 & $\ldots$ & $\ldots$ & 0.143 \\
\hline Iaccarino et al. ${ }^{16}$ & 2-D RANS, $v^{2}-f$ & 0.95 & 2.22 & 0.056 & 1.83 & 0.141 \\
\hline Present & 2-D RANS, SA & 0.95 & 2.26 & 0.37 & 1.13 & 0.139 \\
\hline
\end{tabular}

${ }^{a}$ LES by Porquie, Breuer and Rodi referred to as UKAHY1 in Rodi $e t$ al. ${ }^{22}$

${ }^{\mathrm{b}} \mathrm{RANS}$ by Bosch reported in Rodi et al. ${ }^{22}$

From now on, we examine the flow past a D-shaped cylinder, for which experimental sensitivity results have been documented recently by Parezanović and Cadot. ${ }^{14}$ The cylinder is made of a semicircular leading edge of diameter $D$ continuing into a rectangular aft section, for a combined length of $2 D$. The Reynolds number is $R e=U_{\infty} D / v=13000$, and we compute the time-averaged mean flow repeating the above procedure, including the grid independence tests. All results documented in the following issue from the same mesh $\mathrm{M}_{1}$ built from 182668 triangles (916268 degrees of freedom). In Fig. 1(a), we show a snapshot of vorticity contours evidencing the onset of vortex shedding, found to occur at a frequency $S t_{\mathrm{nl}}=0.23$ consistent with the value $S t_{\exp }=0.22$ measured experimentally for a cylinder of span $H=16 D$ (Ref. 14). In Fig. 1(b), we show the mean vorticity, which turns to be almost perfectly antisymmetric with respect to the centerline. Two shear layers displaying vorticity of opposite signs initiated at the front stagnation point first separate on account of an adverse pressure gradient, reattach on the flat wall and ultimately separate at the sharp trailing edge, hence delimiting a symmetric recirculation bubble of length $\bar{l}_{R}=0.56$. In the inner bubble, negative values of the streamwise velocity reach approximately $40 \%$ of the free-stream velocity. Note that the recirculation length measured experimentally at mid-span is larger $\left(\bar{l}_{R}=0.8\right)$, which could be related to the fact that the near-wake exhibits three-dimensionality, including oblique vortex shedding and vortex dislocations, as reported by Parezanović and Cadot. ${ }^{14}$

We perform a global stability analysis of the turbulent mean flow, and investigate the amplification of small-amplitude superimposed disturbances classically expanded into time-harmonic global eigenmodes $\hat{\boldsymbol{q}}$ of linear growth rate $\sigma$ and eigenfrequency $\omega$, hence reading

$$
\hat{\boldsymbol{q}}(x, y) \mathrm{e}^{\sigma t+\mathrm{i} \omega t}=\hat{\boldsymbol{q}}(x, y) \mathrm{e}^{\lambda t} .
$$

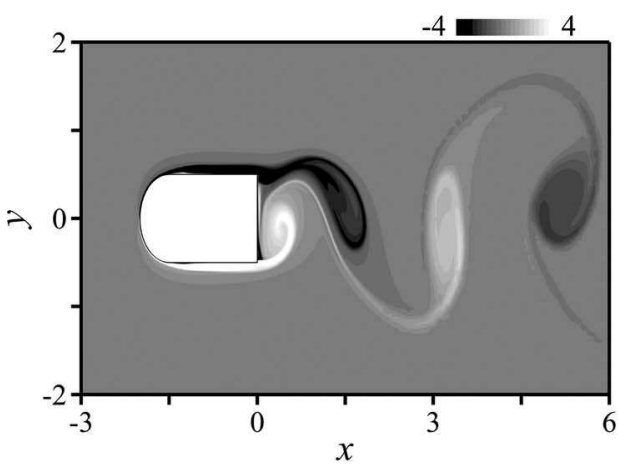

(a)

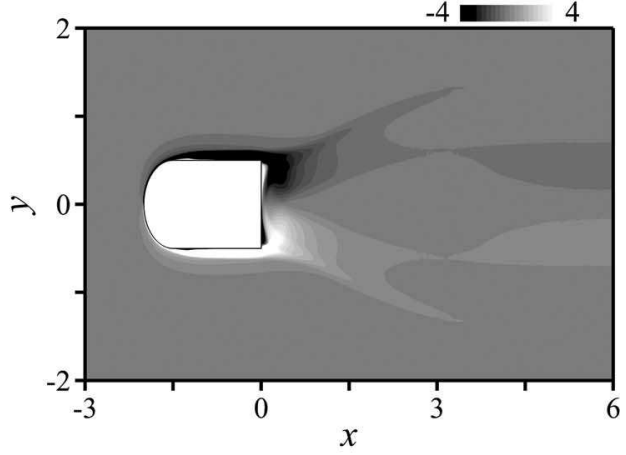

(b)

FIG. 1. 2-D RANS results of flow past a D-shaped cylinder at $R e=13000$. (a) Snapshot of vorticity contours. (b) Contours of mean vorticity. 


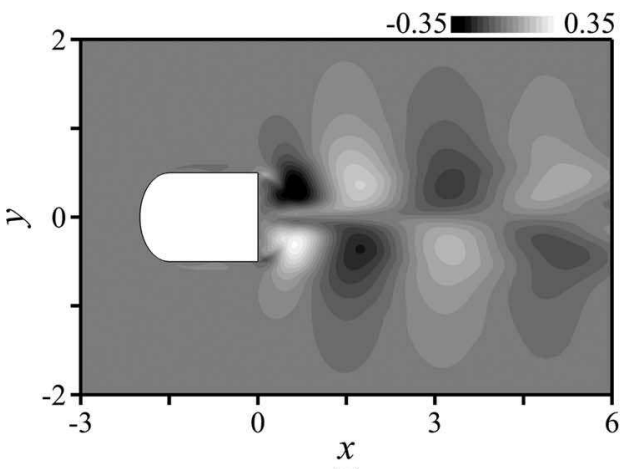

(a)

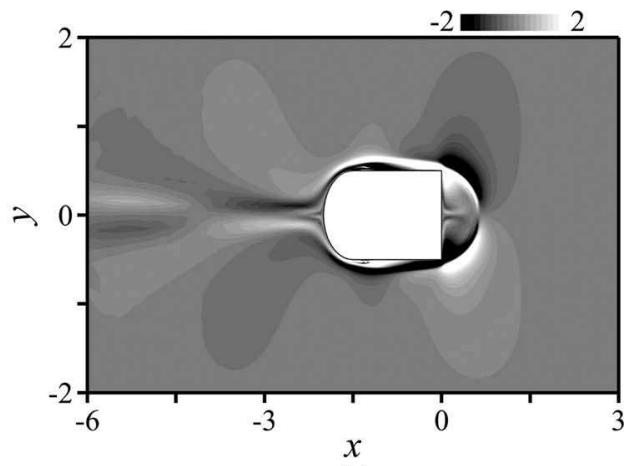

(b)

FIG. 2. Stability analysis of the turbulent mean flow: real part of the streamwise velocity component for the (a) shedding mode and (b) adjoint shedding mode computed linearizing the RANS equations.

The mean flow is viewed here as being rigorously a steady solution of the governing Eq. (1), forced by a source term $\overline{\boldsymbol{F}}=-\overline{\boldsymbol{N}(\boldsymbol{q}, \boldsymbol{q})}$ generated by the large-scale fluctuations associated to vortex-shedding. Following Barkley, ${ }^{9}$ we assume that this forcing can be taken constant to a first approximation, so that the perturbation ultimately satisfies the unsteady RANS equations linearized about the mean flow quantities. This implies especially that all linear results to follow must be properly interpreted as applying in the case where the forcing term $\overline{\boldsymbol{F}}$ is itself unperturbed at the order of the perturbation. As detailed in the supplementary material, we linearize the RANS equations, including the equation for the SA variable, ${ }^{24}$ and compute the eigenpair $\{\lambda, \hat{\boldsymbol{q}}\}$ using the Krylov-iterative solver described in Ref. 19. All boundary conditions are linearized about the mean flow solution, except at the outlet where we apply a stress-free condition. The leading eigenmode, further denoted as the shedding mode, is depicted in Fig. 2(a) by the real part of its streamwise velocity component. It is almost perfectly antisymmetric, with large magnitudes in the shear layers and in the near-wake $(x<4)$, and propagates downstream, as indicated by the real and imaginary parts in spatial quadrature (not shown here). Its eigenfrequency $f=\omega / 2 \pi=0.25$ turns to be a fairly good predictor of the nonlinear Strouhal number. We note yet that the match is not exact: according to Sipp and Lebedev, ${ }^{25}$ this may be due to the fact that a strong resonance occurs with the harmonics of the global mode, which may also explain why the mean flow is presently linearly unstable, the departure from criticality $(\sigma=0.16)$ being comparable to the shift in the eigenfrequency $\left(\omega-2 \pi S t_{\mathrm{nl}}=0.15\right)$. Caution should be used in interpreting these trends since the above study was meant for flow cases close to the bifurcation point, but the argument was already found consistent with the selection of the shedding frequency based on mean-flow stability in turbulent flow past an axisymmetric blunt-based body. ${ }^{11}$ We compute similarly the adjoint shedding mode $\hat{\boldsymbol{q}}^{\dagger}$, i.e., the Lagrange multiplier for the shedding mode, solution of an adjoint eigenvalue problem whose detailed equations are supplied in the supplementary material. The streamwise component depicted in Fig. 2(b) exhibits large magnitudes within the recirculating area, the maximum value being reached close to the separating streamline, but also in the developing boundary layers and upstream of the body, as a result of the nonnormality of the evolution operator. ${ }^{26}$

We now move on to the sensitivity analysis and compute the eigenfrequency variation caused by a small control cylinder of diameter $d \ll D$, positioned at $\left(x_{c}, y_{c}\right)$. Following Hill, we model the presence of the cylinder by a supply of pure momentum equal and opposite to the anticipated drag, hence reading

$$
\boldsymbol{\delta} \boldsymbol{f}(x, y)=-\frac{1}{2} d C_{d}\|\boldsymbol{u}\| \boldsymbol{u} \delta\left(x-x_{c}, y-y_{c}\right),
$$

where $C_{d}$ is a drag coefficient depending on the Reynolds number based on the local velocity and the diameter of the control cylinder. Since the presence of the shedding mode at any arbitrarily small amplitude creates a modulation of the drag vector of same amplitude, the force splits into two distinct 


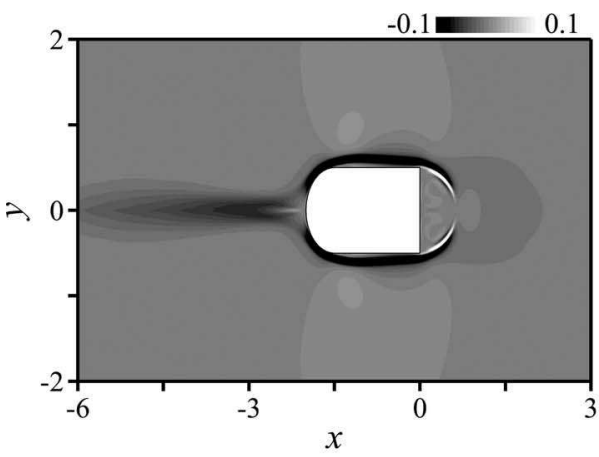

(a)

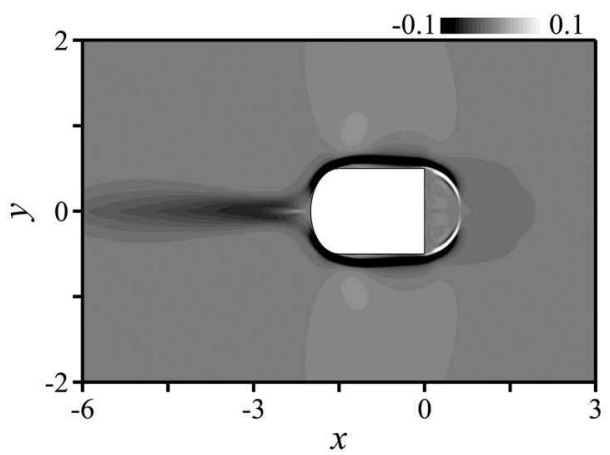

(c)

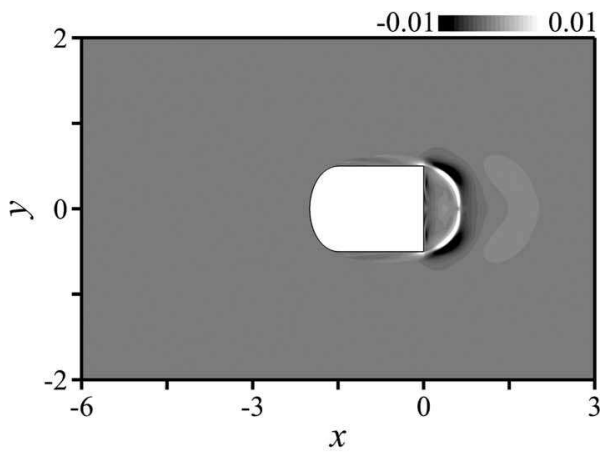

(b)

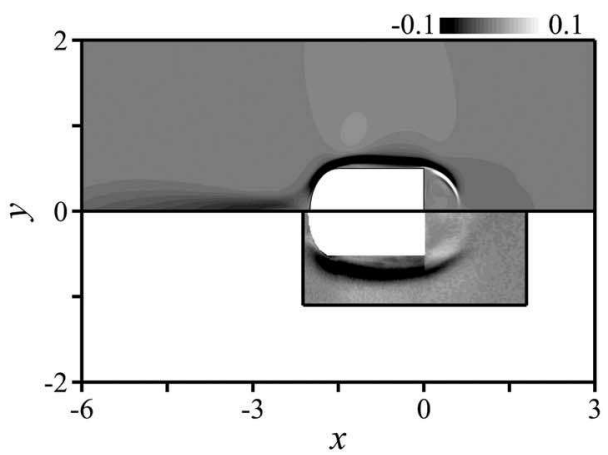

(d)

FIG. 3. Map of the eigenfrequency variation caused by the introduction of a small control cylinder of diameter $d=0.04$. (a) Adjoint-based prediction for the contribution $\left.\delta \omega\right|_{\bar{f}}$ induced by the mean component of drag. (b) Same as (a) for the contribution $\left.\delta \omega\right|_{\hat{f}}$ induced by the harmonic component of drag. (c) Same as (a) for the total variation $\delta \omega=\left.\delta \omega\right|_{\bar{f}}+\left.\delta \omega\right|_{\hat{f}}$. (d) Comparison with the experimental value $2 \pi \delta S t_{\exp }$ of Parezanović and $\mathrm{Cadot}^{14}$ displayed in the lower half of the plot (courtesy of these authors).

components: a steady component $\delta \overline{\boldsymbol{f}}$ forcing the mean flow equations and obtained substituting $\overline{\boldsymbol{u}}$ instead of $\boldsymbol{u}$ in (3), and a harmonic component $\boldsymbol{\delta} \hat{\boldsymbol{f}}$ forcing the stability problem under the form of a feedback force-velocity coupling ${ }^{4}$ and obtained in the quasi-static limit linearizing Eq. (3) about $\overline{\boldsymbol{u}}$, which yields

$$
\boldsymbol{\delta} \hat{\boldsymbol{f}}(x, y)=-\frac{1}{2} d\left[C_{d}\|\overline{\boldsymbol{u}}\| \hat{\boldsymbol{u}}+\left(C_{d}+R e_{d} \frac{\partial C_{d}}{\partial R e_{d}}\right) \frac{\overline{\boldsymbol{u}} \cdot \hat{\boldsymbol{u}}}{\|\overline{\boldsymbol{u}}\|} \overline{\boldsymbol{u}}\right] \delta\left(x-x_{c}, y-y_{c}\right),
$$

with $R e_{d}=\|\overline{\boldsymbol{u}}\| d / \nu$. The quasi-static assumption is expected to hold since the time scale of adjustment of the flow behind the small cylinder is shorter than that behind the large cylinder by at least a factor of the diameter ratio. We ultimately compute the eigenvalue variation as

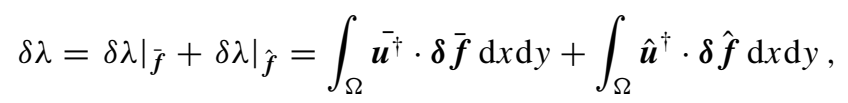

where $\overline{\boldsymbol{q}}$ is a Lagrange multiplier for the mean flow obtained using the adjoint method as the solution of an inhomogeneous linear problem, as explained in the supplementary material.

Figure 3 presents the spatial distribution of the eigenfrequency variation $\delta \omega$ obtained for a diameter $d=0.04$ retaining the imaginary part of Eq. (5). In order to approximate the coefficient $C_{d}$, we fitted a fifth-order polynomial to the steady component of drag tabulated by Fornberg ${ }^{27}$ in the range $R e_{d}<600$ relevant to our flow. The first contribution $\left.\delta \omega\right|_{\bar{f}}$ arising from the mean component of drag, shown in Fig. 3(a), exhibits both negative and positive variations corresponding respectively to a decrease and an increase of the shedding frequency. The frequency decreases placing the control cylinder either inside the shear layers, or upstream of the cylinder, close to the centerline. In contrast, the frequency increases placing the cylinder either in the mean recirculating bubble, or at both sides 


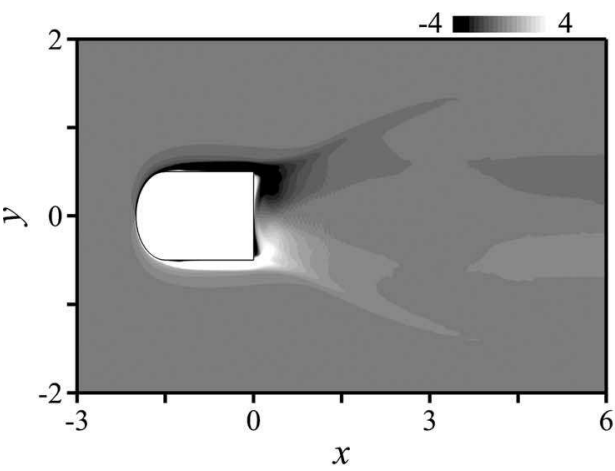

(a)

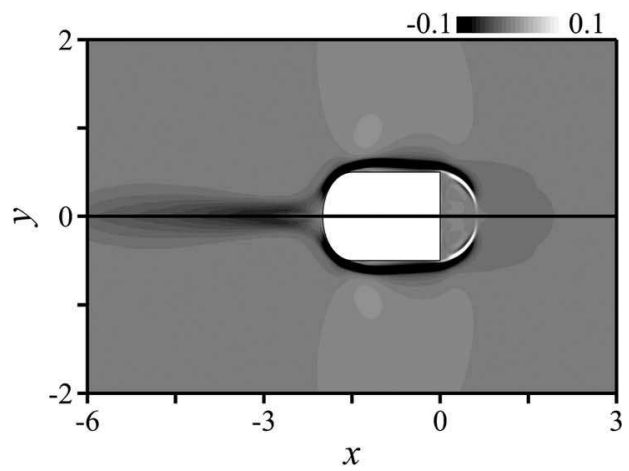

(b)

FIG. 4. (a) Contours of vorticity for the solution $\breve{\boldsymbol{u}}$ obtained by time-averaging the instantaneous solution over one single shedding cycle, starting from $t=100$. (b) Map of the eigenfrequency variation caused by the introduction of a small control cylinder of diameter $d=0.04$. The reference results obtained using the mean flow $\overline{\boldsymbol{u}}$ are reproduced from Fig. 3(c) in the upper half of the plot, while the results obtained using the approximated mean flow $\breve{\boldsymbol{u}}$ are displayed in the lower half.

of the shear layers developing from the upstream stagnation point. The variation $\left.\delta \omega\right|_{\hat{f}}$ arising from the harmonic component, shown in Fig. 3(b), is limited to the vicinity of the mean recirculating bubble. More importantly, it is lower by one order of magnitude and is visible here only owing to an adjustment of the color look-up table. In return, the total variation $\delta \omega$ shown in Fig. 3(c) is entirely dominated by the mean component. We report in Fig. 3(d) experimental measurements of the frequency variation $2 \pi \delta S t_{\exp }$ performed recently at the exact same flow conditions. ${ }^{14}$ The numerical and experimental results are in good qualitative agreement, as the regions yielding either an increase or a decrease of the frequency are especially well reproduced by the theory. Note that the localization and structure of the sensitive regions depend little on the modeling of the drag coefficient $C_{d}$, the difference being only in the magnitude of the achieved variation. Those depicted in Fig. 3(d) compare well with the experiments since the same color code is used to represent both data sets. Our predictions however slightly overestimate the effect of the control, which may be explained noticing that they represent only a linear estimation of the exact eigenfrequency variation, whose calculation would require repeating the mean flow and stability calculations for each cylinder position using expression (3) as a body force to the RANS equations. Note that the experimental and theoretical analyses differ in the sense that the experiments involve only instantaneous measurements of the unperturbed and perturbed flow, for which neither the natural, nor the controlled mean flow solutions need to be determined a priori. Despite the fact that our RANS calculations force the motion to be 2-D, which is not truly representative of the actual experimental flow, as has been mentioned already, the present agreement provides evidence that the control cylinder does act primarily through a 2-D local modification of the mean flow profiles. This is consistent with the finding of Parezanović and Cadot who compared the mean flow structures obtained with and without control cylinder, and thereby correlated the change in the shedding frequency to a modification of the interaction between the detached shear layers. ${ }^{14}$ The validity of our interpretation, however, remains to be discussed for larger diameter ratios likely to induce non-small departure from the natural mean flow structure.

Finally, we repeat the complete analysis using an approximation $\breve{\boldsymbol{u}}$ of the mean flow obtained by time-averaging over one single shedding cycle. Although it contains remnants of the vortex shedding in its downstream part, the approximation is quite good, as seen from the contours of vorticity depicted in Fig. 4(a). It turns to be even excellent in view of the sensitivity analysis: the eigenfrequency variations obtained using $\breve{\boldsymbol{u}}$, depicted in the lower half of Fig. 4(b), are almost identical to those obtained using $\overline{\boldsymbol{u}}$, reproduced from Fig. 3 in the upper half. Moreover, they depend very weakly on the reference time used to start averaging (not shown here). Although it does not avoid the fundamental issue of requiring the vortex shedding solution, the approach eliminates the need to average over a large number of cycles, which may prove important for the foreseeable future since the cost of the overall computations is roughly that of computing the mean flow. In present 
case, it takes less than $15 \mathrm{~h}$ on a regular sequential workstation to obtain the approximated map shown in Fig. 4(b).

In the long run, we aim at applying the methodology to turbulent flows around ground vehicles. Current efforts are thus focused on performing the analysis in the frame of LES, which is known to yield a more accurate representation of those mean flow features (e.g., flow around an Ahmed body), but demands in return substantial modification of the formalism.

The authors are grateful to F. Gallaire for valuable discussions and provision of computational facilities at Laboratory of Fluid Mechanics and Instabilities (Ecole Polytechnique Fédérale de Lausanne, Switzerland), as well as to O. Cadot and V. Parezanović for provision of their experimental data.

${ }^{1}$ P. J. Strykowski and K. R. Sreenivasan, "On the formation and suppression of vortex shedding at low Reynolds numbers," J. Fluid Mech. 218, 71 (1990).

${ }^{2}$ D. C. Hill, "A theoretical approach for analyzing the restabilization of wakes," NASA Technical Report No. 103858 (1992).

${ }^{3}$ A. Bottaro, P. Corbett, and P. Luchini, "The effect of base flow variation on flow stability," J. Fluid Mech. 476, 293 (2003).

${ }^{4}$ F. Giannetti and P. Luchini, "Structural sensitivity of the first instability of the cylinder wake," J. Fluid Mech. 581, 167 (2007).

${ }^{5}$ O. Marquet, D. Sipp, and L. Jacquin, "Sensitivity analysis and passive control of cylinder flow," J. Fluid Mech. 615, 221 (2008).

${ }^{6}$ J. Pralits, L. Brandt, and F. Giannetti, "Instability and sensitivity of the flow around a rotating circular cylinder," J. Fluid Mech. 650, 513 (2010).

${ }^{7}$ P. Meliga, D. Sipp, and J.-M. Chomaz, "Open-loop control of compressible afterbody flows using adjoint methods," Phys. Fluids 22, 054109 (2010).

${ }^{8}$ H. Sakamoto, K. Tan, and H. Haniu, "An optimum suppression of fluid forces by controlling a shear layer separated from a square prism," ASME Trans. J. Fluids Eng. 113, 183 (1991).

${ }^{9}$ D. W. Barkley, "Linear analysis of the cylinder wake mean flow," Europhys. Lett. 75, 750 (2006).

${ }^{10}$ E. Piot, G. Casalis, F. Muller, and C. Bailly, "Investigation of the PSE approach for subsonic and supersonic hot jets. Detailed comparisons with LES and linearized Euler equations results," Int. J. Aeroacoust. 5, 361 (2006).

${ }^{11}$ P. Meliga, D. Sipp, and J.-M. Chomaz, "Elephant modes and low frequency unsteadiness in a high Reynolds number, transonic afterbody wake," Phys. Fluids 21, 054105 (2009).

${ }^{12}$ Y. Hwang and C. Cossu, "Amplification of coherent streaks in the turbulent Couette flow: An input-output analysis at low Reynolds number," J. Fluid Mech. 643, 333 (2010).

${ }^{13}$ M. Marquillie, U. Ehrenstein, and J.-P. Laval, "Instability of streaks in wall turbulence with adverse pressure gradient," J. Fluid Mech. 681, 205 (2011).

${ }^{14}$ V. Parezanović and O. Cadot, "Experimental sensitivity analysis of the global properties of a 2D turbulent wake," J. Fluid Mech. 693, 115 (2012).

${ }^{15}$ W. Rodi, "Comparison of LES and RANS calculations of the flow around bluff bodies," J. Wind Eng. Ind. Aerodyn. 69-71, 55 (1997).

${ }^{16}$ G. Iaccarino, A. Ooi, P. A. Durbin, and M. Behnia, "Reynolds averaged simulation of unsteady separated flow," Int. J. Heat Fluid Flow 24, 147 (2003).

${ }^{17}$ P. R. Spalart and S. R. Allmaras, “A one-equation turbulence model for aerodynamic flows," Rech. Aerosp. 1, 5 (1994), also see AIAA Paper No. 92-0439.

${ }^{18}$ See supplementary material at http://dx.doi.org/10.1063/1.4724211 for provision of the theoretical formalism and detailed equations solved in the paper.

${ }^{19}$ P. Meliga and J.-M. Chomaz, "Global modes in a confined impinging jet: Application to heat transfer and control," Theor. Comput. Fluid Dyn. 25, 179 (2010).

${ }^{20}$ D. A. Lyn, S. Einav, W. Rodi, and J. H. Park, "A laser-Doppler velocimetry study of ensemble averaged characteristics of the turbulent near wake of a square cylinder," J. Fluid Mech. 304, 205 (1995).

${ }^{21}$ B. E. Lee, "The effect of turbulence on the surface pressure field of a square prism," J. Fluid Mech. 69, 263 (1975).

${ }^{22}$ W. Rodi, J. H. Ferziger, M. Breuer, and M. Pourquie, "Status of large-eddy simulation: Results of a workshop," J. Fluids Eng. 119, 248 (1997).

${ }^{23}$ M. Minguez, C. Brun, R. Pasquetti, and E. Serre, "Experimental and high-order LES analysis of the flow in near-wall region of a square cylinder," Int. J. Heat Fluid Flow 32, 558 (2011).

${ }^{24}$ J. D. Crouch, A. Garbaruk, and D. Magidov, "Predicting the onset of flow unsteadiness based on global instability," J. Comput. Phys. 224, 924 (2007).

${ }^{25}$ D. Sipp and A. Lebedev, "Global stability of base and mean flows: A general approach and its applications to cylinder and open cavity flows," J. Fluid Mech. 593, 333 (2007).

${ }^{26}$ J.-M. Chomaz, "Global instabilities in spatially developing flows: Non-normality and nonlinearity," Annu. Rev. Fluid Mech. 37, 357 (2005).

${ }^{27}$ B. Fornberg, "Steady viscous flow past a circular cylinder up to Reynolds number 600," J. Comput. Phys. 61, 297 (1985). 